\title{
An Investigation into the Methods for Analyses and Conservation of Historical Urban Forms
}

\author{
Mert Nezih Rifaioğlu \\ Faculty of Architecture, Department of Architecture, Mustafa Kemal University, Turkey
}

Copyright $(2016$ by authors, all rights reserved. Authors agree that this article remains permanently open access under the terms of the Creative Commons Attribution License 4.0 International License

\begin{abstract}
Historical urban forms are, over time, created out of different and complex tangible and intangible parameters in a continuous and dynamic process that creates a unique set of relations across the urban form. Different methods have been adopted to understand the complex relationships that occur within historical urban forms, and this paper aims to investigate these methods and how they are adopted as part of analysis and conservation efforts. The investigation reveals that the methods applied in urban form studies need to be integrated in order to provide a new methodological framework for historical urban forms.
\end{abstract}

Keywords Historical Urban Form, Integrated
Methodology, Conservation

\section{Introduction}

Different methods are applied for the analysis of urban form that can be grouped under the headings of urban morphological analyses; architectural and urban design analyses; and urban conservation analyses. All such methods have been developed from conventional techniques into their contemporary form with the rapid development of technologies. For example, CAD-CAM programs, SPSS, GIS (Geographical Information System) have begun to be used to great effect in a broad range of fields that include urban morphological studies, architectural studies, and urban design and urban conservation studies.

This research makes an investigation of the typo-morphology method in urban morphological studies; the theory of space-syntax in architectural and urban design studies, and the urban conservation analyses method in conservation studies, and seeks to identify their possible uses with new technological tools. The aim of this investigation is to propose that these methods be put forward as the most suitable for the topic of inquiry, and as a result, other methods, such as interpretivism, ethnography and grounded theory applied by sociologists incorporating in-depth questionnaires, interviews, etc., and the linkage theory and figure-ground theory used by urban designers are not considered in this research in order to limit the study.

\section{Typo-morphological Studies}

Typo-morphological studies have been used throughout history as a design tool, dealing with the physical and spatial structure of the built environment. They are derived from studies of typical spaces and city structures and are based on detailed classifications of buildings and open spaces by type "typology" [1]. In this regard, they consider not only the various scales of the built environment but also the character of the urban form and its inhabitants. As Moudon [1] states, "typo-morphology offers a working definition of space and building type, and serves as a rich launching ground for studying the nature of building design, its relationship to the city, and to the society in which it takes place".

Different methods of inquiry are used in typo-morphological studies. For example, Conzen's method of inquiry relies on three components of the urban form, being street, plot and building; while Muratori's method relies on the history and memory of the urban form; and Rossi's method relies on typology and urban morphology around the themes of typology, function, classification and permanence.

One of the major points of intersection of the different methods of inquiry is their analysis of the built environment within its historical context [1], although the concept of zoning in the historical urban context rises as a fundamental issue in typo-morphological studies. As Kropf [2] states, "zoning ... is a tool for analysis and explanation. It helps us to understand both the physical structure and the historical development of urban areas and the relation between urban areas and individual buildings ... the general structure and mechanism of zoning offer an ideal context for the application of typo-morphological principles ... In particular, a typological approach to zoning addresses the complaint that zoning tends to prohibit historical or traditional forms of buildings and urban fabric".

The principles of typological investigation introduced by 
Italian architect Saverio Muratori, which were developed further by Gianfranco Caniggia and Gian Luigi Maffei, among others, suggest a number of working assumptions for a typological approach to zoning [1]. According to them, zones should be taken as the starting point of local and regional forms; and in multi-cultural historical urban contexts in particular typological studies should be made through zoning, in which should be conceived as tools for the analysis of different built environments in their differentiation between different socio-cultural aspects.

\section{The Theory of Space Syntax}

The theory of Space Syntax was conceived by Hillier and his colleagues in the late $1970 \mathrm{~s}$ as a tool that allowed architects to simulate the likely social effects of their design. They [3] define space syntax as:

"a set of techniques for the representation, quantification, and interpretation of spatial configuration in buildings and settlements. Configuration is defined in general as, at least, the relation between two spaces taking into account a third, and, at most, as the relations among spaces in a complex taking into account all other spaces in the complex. Spatial configuration is thus a more complex idea than spatial relation, which need invoke no more than a pair of related spaces. The theory of 'space syntax' is that it is primarily - though not only through spatial configuration that social relations and processes express themselves in space".

Various measures in urban configuration are correlated consideration of different aspects of social life. In its initial form, space syntax focused mainly on patterns of pedestrian movement in cities, and the method was subsequently extended to include a number of other roles, such as modeling urban traffic, predicting air pollution levels, assessing the occurrence of burglaries in different neighborhoods, and estimating the potential for retail development in streets. Since the 1980s, space syntax techniques have become computer based with the advent of such programs as NewWave 1.0, written by Sheep T. Iconoclast; Axman PPC 2.5, written by Sheep T. Iconoclast, and also a number of other software applications that now exist linking space syntax to GIS in the Space Syntax Laboratory and the Virtual Reality Centre for the Built Environment at University College London.

Over the past decades, with the development of computer based programs, space syntax has come to be applied in such diverse research fields as archaeology, information technologies, urban and human geography, and anthropology, given its obvious reliability in representing and analyzing the social use of space and movement in street networks through axial maps and line/justified $(j)$ graphs.

\subsection{The Axial Map}

The axial map is the most commonly used technique at an urban level, and has been defined by Hillier [4] as:

"In the study of cities, one representation and one type of measure has proved more consistently fruitful than others: the representation of urban space as matrix of the 'longest and fewest' lines, the 'axial map', and the analysis of this by translating the line matrix into a graph, and use of the various versions of the 'topological' (that is, non-metric) measure of patterns of line connectivity called 'integration'."

According to Hillier [4] "... the axial line map generated not only models for predicting urban movement, but also strong theoretical results on urban structure, and even a general theory of the dynamics linking the urban grid, movement, land uses, and building densities in 'organic cities'."

The axial map is based on a simple line representation of the street network, comprising lines that best estimate urban movement. The creation of an axial map begins with the selection of a line (a street or avenue) with a high density in the urban pattern, and then continues with the drawing second, third, fourth, etc. lines that intersect each other. Then, each line on the map is numbered according to how many changes of direction separate it from the start line indicating what is generally referred to as the depth, in a way denoting distance, representing the minimum number of changes of direction required to go from the point/line of origin to any other segment on the network [5].

Unfortunately, these depths cannot be used to compare the status of streets in different cities, as they are affected by the total number of nodes in the system [5]. To take this factor into account, integrated values are used to calculate depths in different places in order to consider local distinctiveness. The level of local vehicle and pedestrian movement through each line is dependent on its integration value. As Hillier states [6], "Integration values in line maps are of great importance in understanding how urban systems function because it turns out that how much movement passes down each line is very strongly influenced by its 'integration value' ... pedestrian densities on lines in local areas can usually be best predicted by calculating integration for the system of lines up to three lines away from each line (radius-3 integration)." 


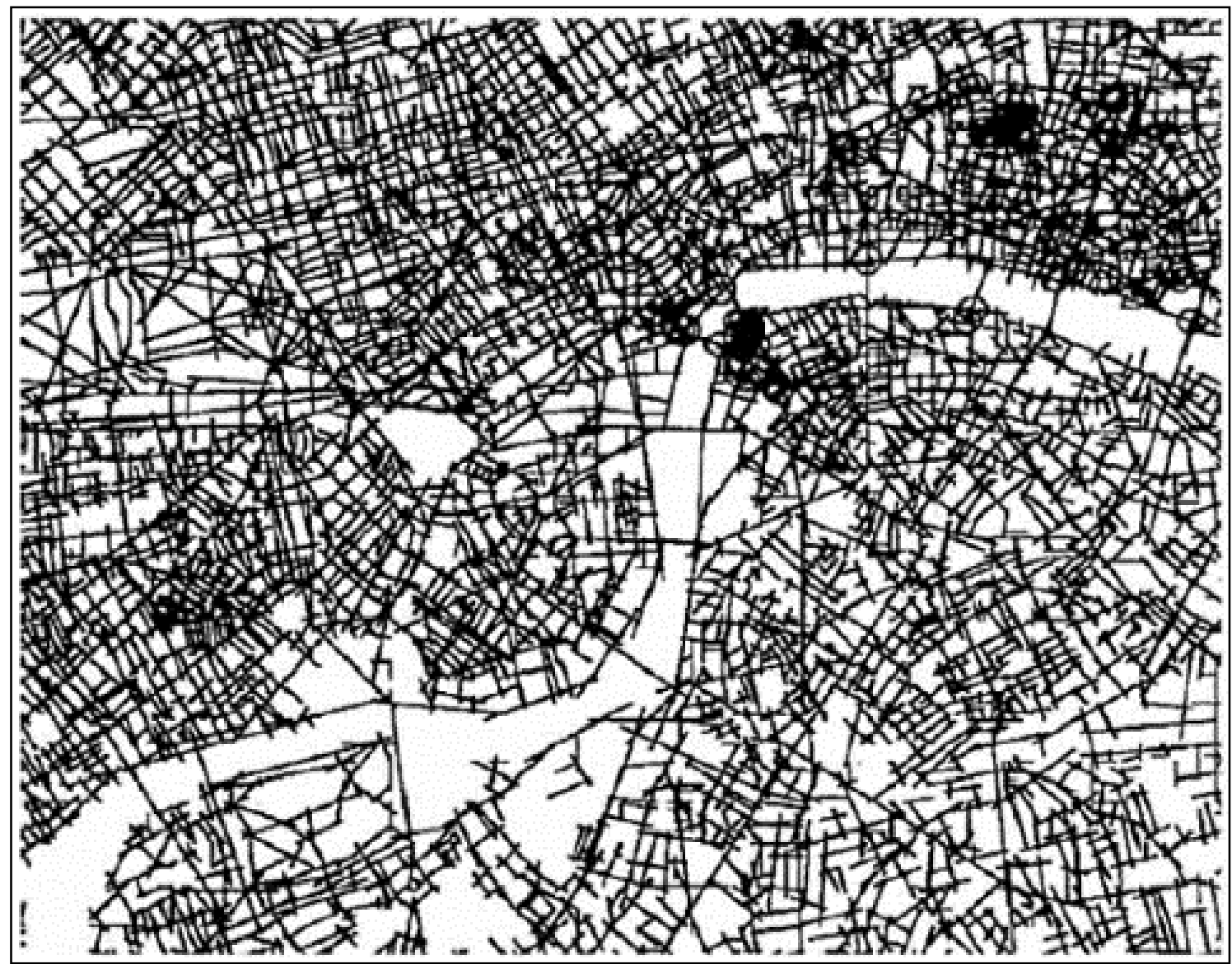

Figure 1. The axial map of London [9]

\subsection{Line/Justified (J) Graphs}

A line/justified (j) graph is logically very similar to an axial map, being used to represent the spatial configuration of buildings, archaeological areas, etc. It is assembled on a 'root' space, with other spaces in the graph aligned above it in levels according to how many spaces one must pass through to arrive at each space from the root [6]. The resulting graphs comprise vertices/nodes and connected links that are referred to as edges/arcs. This method can help in the measurement of the social use of space by articulating the cultural assets, social relations and configurational properties of spatial layouts using a topology that is a branch of mathematics to deal with the properties of spaces as they form connected pieces and have boundaries, independent of their size and shape.

Recently, Carlo Ratti [5], a professor at the SENSEable City Laboratory, MIT (Massachusetts Institute of Technology), has identified some inconsistencies in space syntax, and asks, "how space-syntax possible to tell so many things about the urban environment with such a limited amount of information that is, after having dismissed data such as the height of buildings and the size of streets?" [5]. He claims that the axial map not only ignores all metric information about the city plan and its land uses, but also all 3D information, such as buildings. Aiming to eliminate the inconsistencies in the space syntax method, Ratti [7] and Ståhle et al. [8] proposed the use of DEM (Digital Elevation Modeling) and place-syntax as complementary tools to space syntax for the analysis of urban forms.

\subsection{DEM (Digital Elevation Modeling)}

DEM is widely used in geosciences, and has recently found popularity also in urban studies, becoming indispensable in such analysis forms as topographic feature extraction, slope stability analysis and landscape analysis. There have been a few recent studies hailing its accuracy for built-up area analysis, such as for building heights and bridges, and furthermore, Harvard University and the Massachusetts Institute of Technology have both started using DEM for analyses of local terrain and built-up areas using GIS. 


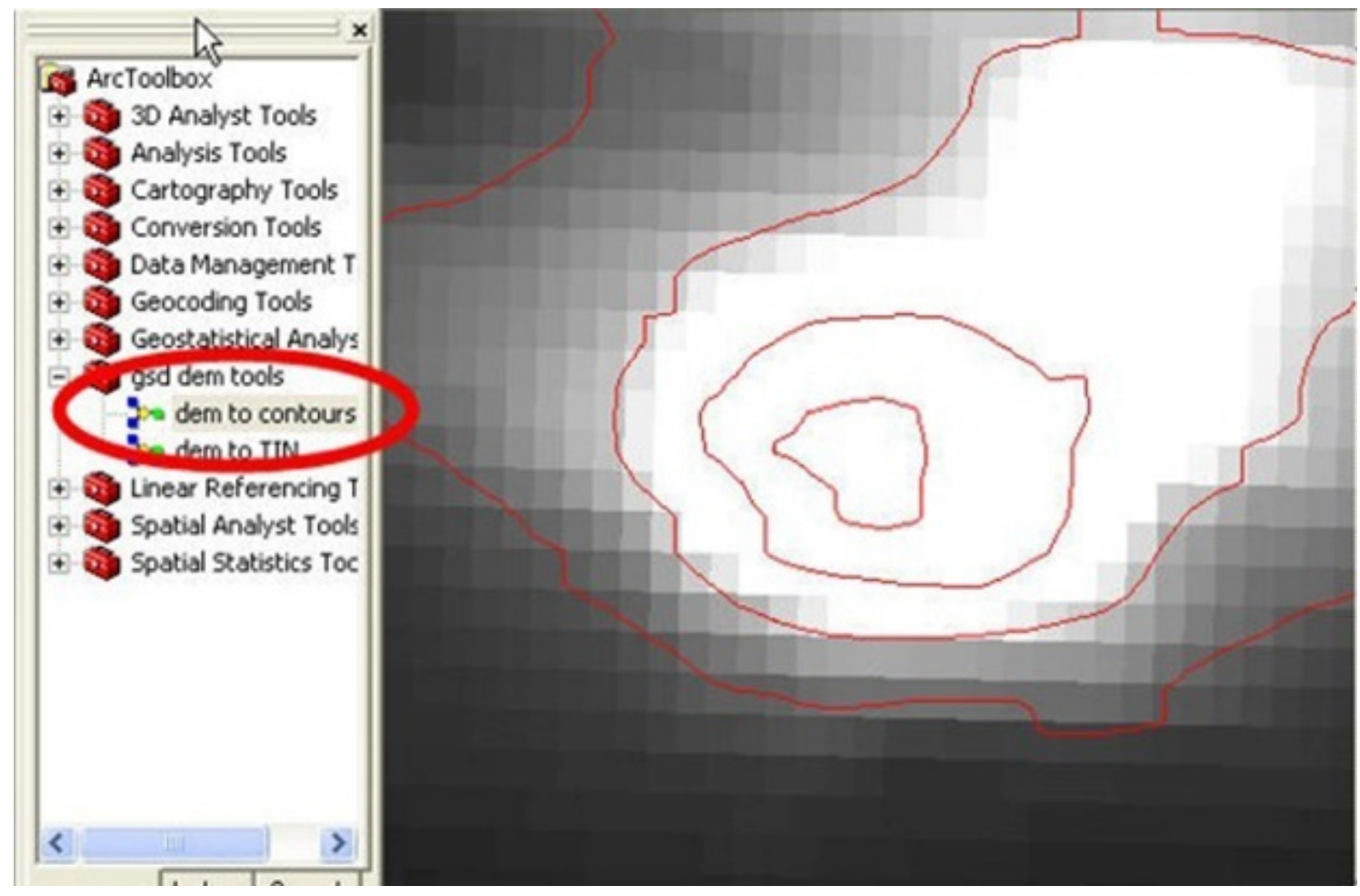

Figure 2. Digital Elevation Models (DEMs) used in a raster layer of GIS [13]

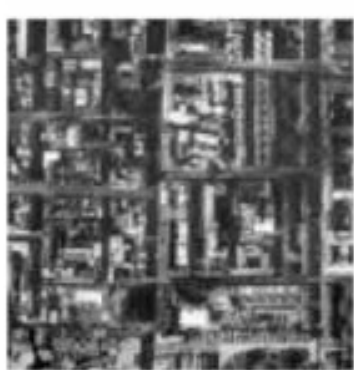

(a)

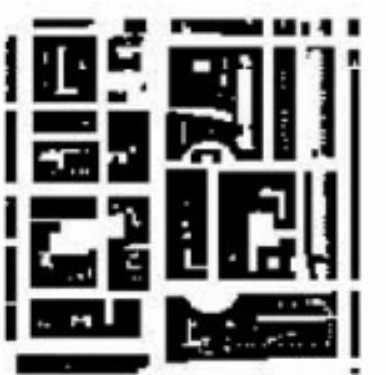

(b)
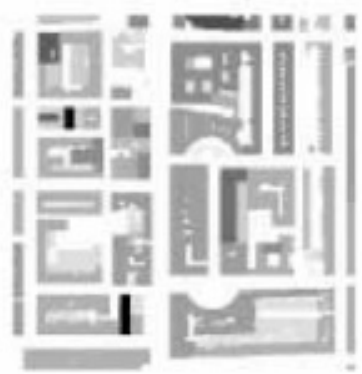

(c)
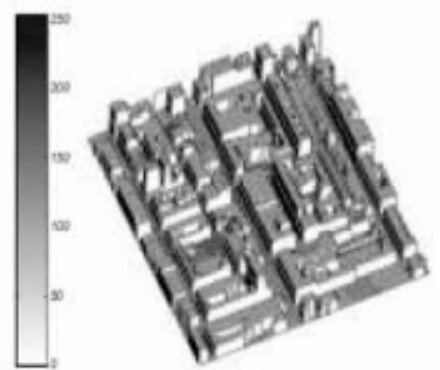

Figure 3. Aerial image of a case-study site in Central London, with (a) a black and white ground map showing built and un built areas (b) and in the digital elevation model format (c) [7].

In the SENSEable City Laboratory (MIT), DEM has since 2004 been used for the analysis of urban forms. Ratti [7] proposes that, "although a simplified format and a concise representation of street networks would probably have been a necessity in the early days of space syntax when computing resources were scarce, it is possible today that a more complete analysis based on a richer support would be helpful to understand the 'social logic of space' ... DEM could effectively complement traditional space syntax in the coming years".

In his study, DEM is used as a raster map for the storage of building heights. He states that, "the DEM is a compact way of storing urban $3 \mathrm{D}$ information using a $2 \mathrm{D}$ matrix of elevation values; each pixel represents building height and can be displayed in shades of grey as a digital image" [7].

Ratti tried to find a possible use for DEM in deciphering 'the social logic of space'. According to him (2005), DEM is a sufficiently appropriate tool both for understanding such 3D components as the height and width of buildings, and 2D components such as visibility analyses and pedestrian movements within the urban form.

\subsection{Place-Syntax}

The place-syntax tool was developed by the Spatial Analysis and Design at Royal Institute of Technology together with the Department of Numerical Analysis and Computing Science in Stockholm, Sweden. Ståhle et al. [8] state that in architectural theory the concept of genius loci has led to concepts that are difficult to handle analytically, and so the place-syntax tool offers great potential for filling the gaps between geographical oriented accessibility research and geometrically oriented research in architecture.

The Place-Syntax Tool is a layer in GIS MapInfo that uses 
spatial data related to plot regions or address points. According to the Royal Institute of Technology Department of Spatial Analysis and Design, the "Place syntax approach and the new GIS-tool has great potential for urban analysis and architectural space design, for example, in better predicting pedestrian flow and estimating accessibilities based on the experiencing subject, both where the pedestrian can go and what it can see" [9].

The papers entitled "Place Syntax: geographic accessibility with axial lines in GIS" by Ståhle, Marcus \&Karlström and two poster papers entitled "Park Syntax" and "Plot Syntax" delivered at the 5th International Space Syntax Symposium 2005 in Delft all stressed the potential uses of place syntax for the analysis of urban from as a complimentary tool to space syntax. Although it seems to be useful, it is still developing as a tool, and to date there have been no other publications demonstrating the use of place syntax in the urban form.

\section{Urban Conservation Analyses}

Understanding and appreciating an area's identity, including its social and economic background and the way in which such factors have shaped the urban fabric, should be the starting point when making decisions about both its management and its future [10]. In this regard, to keep and preserve the identity in urban conservation, adopting a well-defined urban conservation analyses method is essential.

In the designation of conservation areas, effectiveness against globalization requires the making of rational and consistent judgements when determining their special qualities and local distinctiveness, and such designations should be based on a thorough understanding of the area in its wider context, based on a detailed appraisal of its character [10].

Accordingly, objectivity becomes an important factor in the forming of rational and consistent judgements related to the designation of conservation areas. Different stakeholders, including social groups, political groups, governmental and non-governmental organizations, occupants, etc. play an important role in such an approach, fostering the making of objective decisions related to conservation areas. As Kong [11] stresses:

"Urban forms result from and contribute to the conflicts between different value systems ... Such value systems reflect the interests of different social, economic, and political groups, whose divergences take shape variously as contestation between state and civil society, for example, between urban planners and heritage groups, or tourism planners and local interest groups. Such value conflicts shape the different identities that different groups construct for their cities. At the risk of oversimplification, it may be said that pro-development groups are concerned with developing a city that is characterized by modernity, while pro-heritage groups are more enamored by cities identified with history, culture, and other symbolic values."

Elaborating Kong's definition of objectivity, Kropf [12] brought two new concepts to the table in understanding and appreciating the identity/character of towns, being the perspectives of the "observer" and the "observed". Observers are experts who should observe the historical urban context with objective tools in order to properly designate and conserve the local distinctiveness of towns. In this regard, observers in an urban conservation analysis should be part of a collaborative study, and should have expertise in different disciplines such as conservation, architecture, city planning, archaeology, history, engineering, etc. through the observed, being the town itself. Accordingly, the methodology used in a conservation analysis should consider the various actors and aspects of the town itself through an interdisciplinary approach, and such a methodology, applied in all the stages of an urban conservation analysis, is the main tool for the identification and conservation of local identities in most countries. This approach includes a careful and systematic documentation, analysis and evaluation of the data related to socio-cultural, economical, administrative, historical, and physical aspects of urban contexts at various scales, contributing to the making of correct decisions for their conservation.

Table 1. The main aspects and scales in an urban conservation analysis

\begin{tabular}{|c|c|}
\hline ASPECTS & SCALES \\
\hline Socio-Cultural & Region \\
\hline Physical & Settlement \\
\hline Economic & Environmental \\
\hline Historical & Zone \\
\hline Administrative & Building \\
\hline
\end{tabular}

In order to understand and appreciate the historical urban context in urban conservation projects, various technological tools are used, besides the more conventional ones. For example, AutoCAD is used for the preparation of architectural drawings and for the design of project areas; GIS (Geographical Information System) is used for the establishment of a spatial information system that allows for the storage, structuring, analysis, assessment and monitoring of all the spatial data; and a RealVIZ Image Modeler is used to provide rectified photos of all buildings on the site.

In order to understand and appreciate the historical urban context, urban conservation projects begin with a documentation phase comprising a pre-survey and survey. The pre-survey includes a comprehensive literature review, during which all graphic, historical, verbal and written documents related to various aspects of the site are gathered. Then, base maps at various scales and survey sheets related to the buildings and social groups are prepared that can be used in the collection of data during the field work. In this regard, the maps and data sheets assist in the gathering of 
required information on the general characteristics of the site, providing a foundation for the subsequent stages [13].

During the survey stage, data related to socio-cultural, economic, administrative, historical and physical features at building, site, environment and settlement scales are collected using survey sheets and social questionnaires. Such a systematic method in a site survey leads the observer to document the various dimensions and components of the urban context, including information about open and built-up areas, open public spaces, infrastructure and public services, traffic-circulation, natural elements, private open areas, landmarks, vista/panorama points, nodes, preserved building groups, socio-cultural activities, customs of inhabitants, economic activities, touristic and historical places and/or viewpoints, building spaces, architectural elements, materials, structural and construction techniques, deterioration, changes, the characteristics and attitudes of the inhabitants towards the site, historic buildings, and so forth [13].

The data collected during the pre-survey and survey stages is analyzed and evaluated in the subsequent stages. In the evaluation stage, the general characteristics of the site, as well as its values, problems and potentials are defined based on objective criteria, providing a deep understanding and appreciation of the town and the classification of its two- and three-dimensional components that are to be to be protected [13].

In the final decision stage, several details should be taken into consideration in order to ensure an integrated, sustainable, and manageable conservation process. To this end, decisions on the legal, administrative, physical, social, cultural and economic aspects of the site; the types and degrees of interventions; and specific decisions related to the preservation, rehabilitation and re-functioning of building groups are defined [13].

\section{Conclusions}

Methods for the analysis and conservation of historical urban forms have been established over time with the strong catalyst of technology in the form of new software and tools for the analysis of complex urban forms in analytical ways.

Considering the above discussions and the available tools for the consideration of the requirements of each individual town, two questions come to light on this issue:

1. Is it possible to define an urban conservation analysis methodology that can provide a better framework both for the observers and decision makers when dealing with urban conservation projects?

2. Can space-syntax with DEM and place-syntax tools and typo-morphological studies be adopted as supplementary methods to urban conservation analyses in a bid to understand the social, cultural and physical features of towns in analytical ways?

In the light of these discussions and questions, it is argued that, for the analysis and identification of local identities of the historical urban context and the definition of their characteristic aspects in a rational way, an integrated methodology that comprises methods of urban conservation, typo-morphology and space-syntax can be developed and used in the preparation of urban conservation projects. The main aim in this approach is to provide the means of analyzing the historical urban context in detail in which the potential of each method is increased by maximizing their strengths and minimizing their weaknesses.

As mentioned previously, urban conservation analyses comprise four main stages, namely pre-survey, survey, analysis and evaluation, and decision. The pre-survey stage can utilize the typo-morphology method to define the zones based survey through the historical urban context, in that zoning can help in the understanding of both the physical structure and historical development of the historical urban context in relation to its urban areas and individual buildings. To this end, the pre-survey stage should take the form of an analysis of the streets and blocks, plots, building types and land uses through the use of archive materials, graphics, verbal and written materials, historical maps, cadastral maps, and base maps in 1/5000,1/1000 and 1/500 scales in order to identify and describe the distinct characteristics of each zone. After this, the data can be collected for the survey stage through the use of survey sheets compiled during an urban conservation analysis charting the distinct characteristics of each zone.

In the analysis stage, besides the main urban conservation analyses, space syntax can be used to analyze the current use of spaces in traditional buildings, such as the syntax of the ground floors, courtyards, and semi-private and public open areas, and to define the street network to provide an understanding of social movements through the town itself. It can also help in uncovering the social logic behind the formation of spaces and urban areas within the historical urban context. In addition, the typo-morphology method can be used in the analysis stage to define building-lot, façade, plan, architectural element, etc. typologies. By using GIS both in space-syntax with DEM and urban conservation analyses, the data generated by the space-syntax and conservation methods can be integrated and analyzed easily in the evaluation stage.

It is argued that by taking into consideration of the requirements of each town, such an integration methodology can provide a framework both for observers and decision makers when dealing with urban conservation projects, in that it can provide an understanding and the means of analysis of the social, cultural and physical features of a town. As urban conservation projects are recognized as an effective tool in the definition of a town's unique characteristics, their objectiveness and comprehensiveness become important, and so should take into account of the socio-cultural, economic, administrative, historical and physical features of towns at both regional and settlement scales, as well as at a building scales, if they are to fully appreciate the local identity of a historical urban context. 


\section{REFERENCES}

[1] A.V. Moudon. Getting to Know the Built Landscape: Typo morphology. In Franck, K. A. Schneekloth (Eds.), Ordering Space, New York, pp.289-311, 1984.

[2] K. Kropf. Typological Zoning. In Petruccioli, A. (Ed.), Proceedings of the International Symposium on Typological Process and Design Theory, (pp.127-141). Cambridge: MIT Press, 1998.

[3] B. Hillier, J. Hanson, H. Graham. Ideas are in things: an application of the space syntax method to discovering house genotypes. Environment and Planning B: Planning and Design. 14, 363-385, 1987.

[4] B. Hillier. The hidden geometry of deformed grids: or, why space syntax works, when it looks as though it shouldn't. Environment and Planning B: Planning and Design, 26 169-191, 1999.

[5] C. Ratti. Space syntax: some inconsistencies. Environment and Planning B: Planning and Design, 31, 487-499. Retrieved March 24, 2007, from EBSCOhost Academic Search Premier Database, 2004.

[6] B. Hillier. Space is the Machine: A Configurational Theory of Architecture, Cambridge: Cambridge University Press, 1996.

[7] C. Ratti. The lineage of the line: space syntax parameters from the analysis of urban DEMs. Environment and Planning B: Planning and Design, 32, 547-566. Retrieved March 24, 2007, from EBSCOhost Academic Search Premier Database, 2005.

[8] A. Ståhle, L. Marcus, A. Karlström. Place Syntax - Geographic Accessibility with Axial Lines in GIS. 5th International Space Syntax Symposium, Delft. Retrieved May 12, 2007, from (http://www.arch.kth.se/sad/), 2005.

[9] http://www.arch.kth.se/sad/

[10] English Heritage. Guidance on Conservation Area Appraisals, English Heritage, London, 2006.

[11] L. L. Kong. Value Conflicts, Identity Construction, and Urban Change. In Le Gates, R., Stout, F. (Eds.), (pp. 354-365). The City Reader, London, 2000.

[12] K. Kropf. Urban tissue and the character of towns. Urban Design International, 1(3), 247-263, 1996.

[13] M.N. Rifaioğlu. N. Şahin Güçhan. The Concept of Identity and Its Identification Process in Urban Conservation Projects. CSAAR on Regional Architecture and Identity in the Age of Globalization International Conference. pp. 1099-111, Tunis, 2007. 